\title{
Exploring nutrition knowledge and the demographic variation in knowledge levels in an Australian community sample
}

\author{
Gillian Anne Hendrie ${ }^{1,2, *}$, John Coveney ${ }^{1}$ and David Cox ${ }^{2}$ \\ ${ }^{1}$ Department of Public Health, Flinders University, Adelaide, Australia: ${ }^{2}$ CSIRO Human Nutrition, \\ PO BOX 10041, Adelaide BC SA 5000, Australia
}

Submitted 16 September 2007: Accepted 3 April 2008: First published online 1 August 2008

\begin{abstract}
Objectives: Explore the level of general nutrition knowledge and demographic influences of knowledge levels in a community sample.

Design and setting: A sample of volunteers, recruited from community centres in two suburbs of differing socio-economic status, in Adelaide, South Australia.

Subjects: Two hundred and one people, aged 18 years and older, completed a modified and validated version of the General Nutrition Knowledge Questionnaire (113 items). The questionnaire was self-administered and completed under supervision.

Results: Basic messages about eating more fruit, vegetables and fibre, and less fatty and salty foods were best understood. Confusion was evident with more detailed nutrition information. For example, $90 \%$ of the people were aware of the recommendations to eat more fruit and vegetables, but $56 \%$ and $62 \% \mathrm{knew}$ the recommended number of servings of fruit and vegetables, respectively. Descriptive statistics showed significant demographic variation in nutrition knowledge levels; multiple regression analysis confirmed the significant independent effects of gender, age, highest level of education and employment status on nutrition knowledge level $(P<0.01$ level). The model accounted for $40 \%$ of the variance in nutrition knowledge scores.

Conclusions: There is demographic variation in nutrition knowledge levels and a broad lack of awareness of some public health nutrition recommendations. Having a detailed understanding of the deficiencies in community knowledge should allow for future nutrition education programmes to target subgroups of the population or particular areas of nutrition education, to more efficiently improve knowledge and influence dietary behaviour.
\end{abstract}

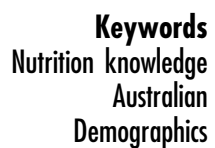

Nutrition knowledge is necessary although not sufficient for dietary change. In line with the Social Cognitive Theory ${ }^{(1)}$, knowledge is one of several factors required to change behaviour, and the significance of knowledge needs to be investigated in terms of its impact on dietary behaviour. An underlying assumption of nutrition education is that increasing knowledge will change behaviour ${ }^{(2)}$.

A meta-analysis conducted in the mid-1980s included nine studies and provides support for this relationship between nutrition knowledge and adopting 'healthier' dietary behaviours ${ }^{(3)}$. More recent studies also maintain that nutrition knowledge is an influential factor of favourable dietary-related behaviour, be it in terms of cholesterollowering dietary approaches ${ }^{(4)}$, compliance with nutrition messages ${ }^{(5)}$, purchasing of healthier foods ${ }^{(6)}$, consumption of lower-fat diets ${ }^{(7-9)}$, consumption of more fruit ${ }^{(10)}$ and vegetables $^{(11)}$, healthy eating in general $^{(12)}$ or greater weight loss ${ }^{(13)}$.

Measuring nutrition knowledge and food-related behaviour is difficult, particularly in a community setting because knowledge forms part of a dynamic cluster of behaviours mediating dietary intake. Worsley ${ }^{(14)}$ suggests that knowledge can be taught, but how consumers interpret and use the information may be difficult to capture as the behavioural response may be delayed. Some of the strongest support for the importance of knowledge as a mediator of dietary intake has come from comprehensive assessments of individuals' nutrition knowledge. Wardle et al. ${ }^{(9)}$ used a broad 110 -item questionnaire covering a range of nutrition concepts, and found individuals with higher knowledge levels were almost twenty-five times as likely as those with little understanding to meet fruit and vegetable intake recommendations and consume a diet lower in fat.

Public health media campaigns are an effective way to change community knowledge and related behaviour. An enduring nutrition message has been the relationship between fat, heart disease and weight control. The National Heart Foundation of Australia, for example, has been disseminating information about heart disease 
throughout the community since the late 1950s. In a national community survey conducted in 1990 ( $n$ 916), two-thirds of the respondents were aware of the association between fat and heart disease and 45\% were aware of the association between $\mathrm{Na}$ and hypertension ${ }^{(15)}$, but measuring the direct effects of the National Heart Foundation's campaigns on behaviour is more difficult.

A more recent public health nutrition education programme in Australia is the Western Australian Health department's Go for $2 \& 5^{\circledR}$ campaign $(2002-2005)^{(16)}$. This multi-strategy fruit and vegetable campaign included media advertising and, importantly, an ongoing evaluation process. The simple nutrition message was well received by the community with about $90 \%$ of the people able to recall the recommendation for fruit and $47 \%$ for vegetables, 12 months after the campaign. With a discrete outcome measure, servings of fruit and vegetables consumed, the effectiveness of the campaign in influencing behaviour could be measured. There was a population net increase of $0 \cdot 8$ in the mean number of servings of fruit and vegetables per day over the 3-year campaign period $^{(18)}$. Following this success, the campaign was rolled out nationally with a similar positive influence on behaviour change ${ }^{(17)}$.

Understanding knowledge and the influences of knowledge within the community is critical in developing dietary behaviour change strategies. Demographic characteristic are associated with nutrition knowledge levels. Females have been shown to have greater nutrition knowledge than males ${ }^{(12,15,18-20)}$. Nutrition knowledge has been shown to increase with age ${ }^{(18)}$, and with an increasing level of formal education ${ }^{(4,18,20-22)}$. There is some evidence to support the relationship between nutrition knowledge and socio-economic status (SES). Studies suggest a link between a higher SES and increased levels of nutrition knowledge ${ }^{(12,18,19)}$.

The primary aim of the present study was to explore in detail the level of general nutrition knowledge within a South Australian community sample using an instrument of known validity and reliability, and second to examine the demographic variation in knowledge levels.

\section{Methods and materials}

\section{Participants}

Participants were volunteers from community centres within two different South Australian local government council areas. Because SES is thought to be a predictor of nutrition knowledge, the objective of recruitment was to establish a sample from areas of differing SES. The Australian Bureau of Statistics has developed indices to allow ranking of areas to reflect social and economic well-being. The Index of Disadvantage, one of four indexes in the Socio-Economic Indexes for Areas (SEIFA), is derived from attributes such as income, educational attainment and unemployment in areas. In the present study, area A was considered to be of a 'low SES', ranked lowest by the Index of Disadvantage in the Adelaide Statistical Division, and area B, 'middle SES', was ranked 8 th of $19^{(23)}$. Participants were attending non-healthrelated groups at the community centres and were approached by the centre organiser to participate in the study. The group members then volunteered to complete the questionnaire. The study was approved by the Flinders University Social and Behavioural Research Ethics Committee and all participants gave informed consent.

\section{Questionnaire and administration}

Nutrition knowledge was measured using a modified and recently validated version ${ }^{(24)}$ of the General Nutrition Knowledge Questionnaire ${ }^{(25)}$. The questionnaire was selfadministered and completed at the community centre under supervision. G.A.H. attended the normal group session and administered the questionnaire to willing group members who completed it without discussion or assistance. The questionnaire contained 113 items relating to four areas of nutrition knowledge: dietary recommendations (thirteen items), sources of nutrients (seventy items), choosing everyday foods (ten items) and the diet-disease relationships (twenty items). Correct responses from each section were added to give a section score, and the four section scores were totalled to give an overall knowledge score out of 113. A higher score reflects a higher knowledge level.

The respondents answered on a range of different scales such as 'more, same, less, don't know', 'yes, no, not sure', 'high, low, not sure', 'agree, disagree, not sure' or a choice of four different food options. Some items required written responses. Demographic questions of gender, age, martial status, number of children, highest level of education and employment status were included at the end of the questionnaire.

\section{Statistical analysis}

Raw data were coded numerically and entered into the computer. Knowledge sub-scores and an overall nutrition knowledge score were calculated. Descriptive statistics were used to analyse the demographic information. Univariate analysis was used to examine the effect of demographic characteristics on nutrition knowledge levels and simultaneous multiple regression analyses were used to explain the variance in nutrition knowledge levels within the sample. Data were entered and analysed using the Statistical Package for Social Sciences Statistical Software package version 11.0 (SPSS Inc., Chicago, IL, USA).

\section{Results}

\section{Study sample characteristics}

Of the 201 people who completed the questionnaire, 105 (52\%) were from area A, the lower SES council area. 
The majority of the sample were female (85\%), and their ages ranged from 18 to 74 years. Table 1 presents the demographic characteristics of the study participants.

What follows is a general description of the research findings, followed by a breakdown of the results in relation to sociodemographic variables.

\section{Awareness of current dietary recommendations}

This part of the questionnaire assessed knowledge of the Australian dietary guidelines and other public health nutrition messages. The mean score was 8.89 (SD 1.94) out of a possible 13 . The nutrition messages of eating more fruit and vegetables, less sugary, fatty and less salty foods were understood by the majority of the community, with $90 \%$ or more of respondents aware of the correct recommendations. There was some confusion about the detailed recommendations for lean meat, high complex carbohydrate foods and low-fat dairy products. For example, $96 \%$ of the respondents were unaware of the recommendation to consume more complex carbohydrate foods. Most people knew to cut down on saturated fat (79\%) and consume low-fat dairy products (69\%). Regarding the current recommendations of consuming two servings of fruit and five servings of vegetables, $56 \%$ and $62 \%$ of the respondents, respectively, were aware of these recommendations.

\section{Knowledge of food sources of nutrients}

There was a maximum score of 70 in this section and the mean in this sample was $43 \cdot 23$ (SD 12.73). Respondents were best able to identify food sources high or low in added sugar, salt and protein, and less able to identify foods high or low in fat, saturated fat and fibre. Respondents also found it difficult to identify healthy alternatives to red meat and foods high in carbohydrates. Despite the general confusion about foods high in carbohydrates, $75 \%$ of the respondents could identify from a list of bread types that wholegrain bread contained the most vitamins and minerals.

A number of specific questions about food sources of nutrients were poorly answered. Seventy-five per cent of people were unaware that fat is the most energy-dense macronutrient. About two-thirds of the sample failed to recognise that butter and margarine are similar in energy and that dairy products are a source of saturated fat. Over half the sample (54\%) believed that brown sugar was a healthier alternative to white sugar.

\section{Making everyday food choices}

This section, identifying a healthy food choice, had a mean score of $6 \cdot 03$ (SD $2 \cdot 13$ ), out of a possible 10 . Respondents were best able to select a low-sugar option from a list of four snack food alternatives, and least able to select a lower fat cheese from a list of four. There was uncertainty in two questions referring to mixed meal proportions of carbohydrates and protein. For example, one question refers to the healthiness of two thick slices of bread and a thin slice of cheese compared to two thin slices of bread and a thick slice of cheese - and 59\% of people answered correctly, choosing the thicker slices of bread. The listed food choices appeared to affect the responses. Most respondents (73\%) were familiar with baked beans on toast as a high-fibre meal; however, less than half (48\%) thought sultanas were a high-fibre snack.

\section{Diet-disease relationships}

Out of a maximum of 20 points, the mean score for this section addressing the diet-disease relationships was $7 \cdot 79$ (sD 3.47). Respondents were most familiar with the relationship between the amount of fat in the diet and disease. Three-quarters reported that this relationship was with heart disease or obesity, but only one quarter knew it was with both heart disease and obesity. Similarly, over $80 \%$ of people were aware of a relationship between the amount of sugar in the diet and disease, but almost all believed that too much sugar directly increases the risk of diabetes. Approximately two-thirds of the sample acknowledged a relationship between the intake of fruits and vegetables, fibre and $\mathrm{Na}$ and disease; however, knowledge of specific diseases was poorly understood. For fruit and vegetables, the most commonly mentioned diseases were bowel disease and scurvy. Many people reported $\mathrm{Na}$ intake to be related to heart disease in general but only $24 \%$ mentioned elevated blood pressure specifically.

About seven out of ten people (69\%) correctly associated eating more fruit and vegetable and more fibre with a reduced risk of cancer, but the same proportion also believed eating less preservative and additives would also reduce the risk of cancer. Seventy-two per cent of respondents correctly identified a diet lower in saturated fat and salt and higher in fruit and vegetables as being protective against heart disease but about two-thirds also thought more fibre and less preservatives was protective.

Almost $70 \%$ of the respondents had heard of the term 'antioxidant vitamins' but most were unsure as to which vitamins were classified as antioxidants. When asked to identify which vitamins have antioxidant properties from a list, less than one-third gave the correct answer on any one vitamin and only $14 \%$ correctly identified vitamins A, $\mathrm{C}$ and $\mathrm{E}$ as antioxidants.

\section{Demographic variation in nutrition knowledge}

\section{Descriptive statistics}

The sociodemographic variation in nutrition knowledge is presented in Table 1. Statistical analysis shows that females scored higher in two of the four knowledge sections and in the overall knowledge score females $(\mu=67 \cdot 02$, SD $17 \cdot 52)$ also scored significantly higher than males $(\mu=59 \cdot 77$, SD $18 \cdot 72)$. Nutrition knowledge increased with age. Participants aged 35 years and older scored significantly higher knowledge levels across each 
Table 1 Univariate analysis of demographic variance in nutrition knowledge

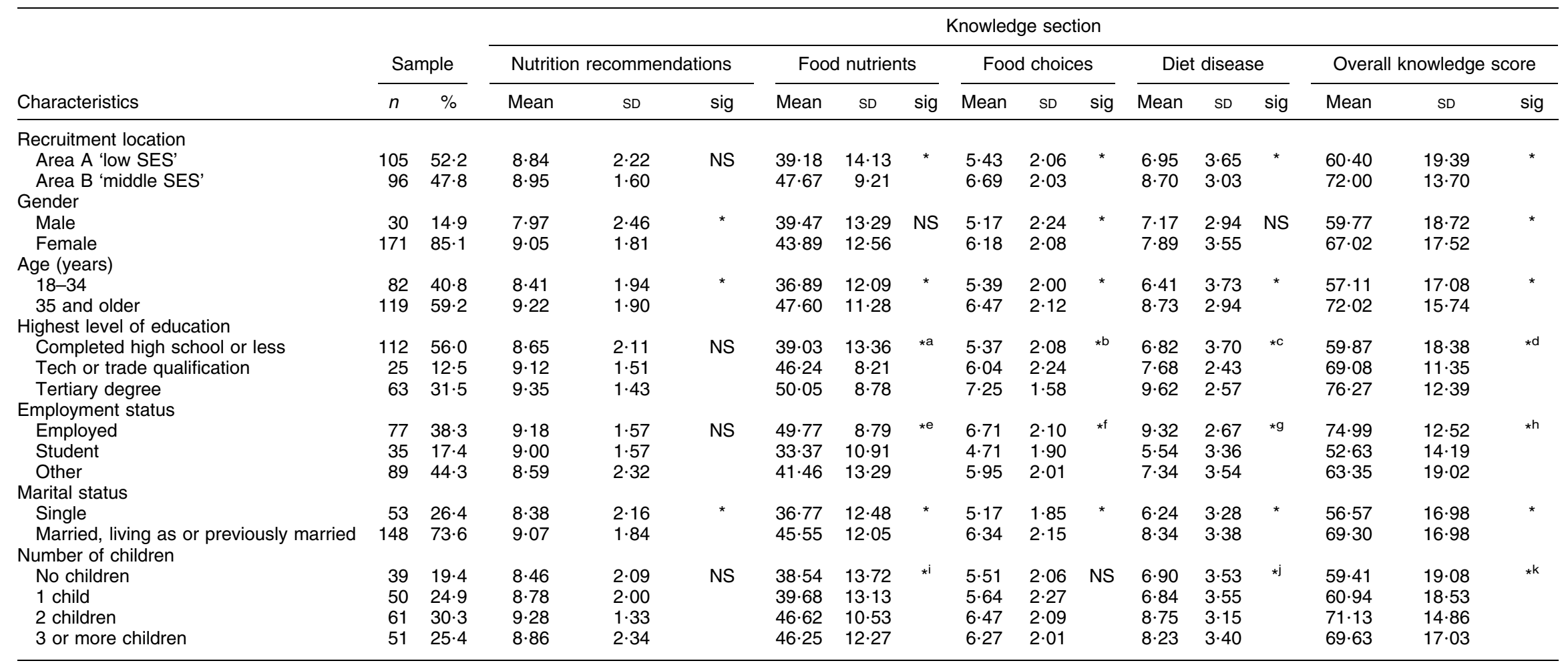

sig, significance; SES, socio-economic status.

a 'Completed high school or less' has significant lower knowledge level than other education categories.

b,c,d 'Tertiary education' has significantly higher level of knowledge than other education categories.

$\mathrm{i}, \mathrm{j}, \mathrm{k} \cdot \mathrm{A}$. 
Table 2 Multiple regression analysis of nutrition knowledge on age, employment status, education and gender

\begin{tabular}{lccc}
\hline & & Nutrition knowledge & \\
\cline { 2 - 4 } & Unstandardised $\beta$ & Standardised $\beta$ & $P$ value \\
\hline Age group & $4 \cdot 028$ & 0.369 & $0 \cdot 000$ \\
Employment status & $3 \cdot 183$ & $0 \cdot 266$ & $0 \cdot 000$ \\
Education level & $3 \cdot 607$ & $0 \cdot 262$ & $0 \cdot 000$ \\
Gender & $8 \cdot 168$ & $0 \cdot 167$ & $0 \cdot 003$ \\
& Multiple $R=0.642 ;$ Adjusted $R^{2}=0.400 ; F(4,195)=34 \cdot 170 ; P=0 \cdot 000$ \\
\hline
\end{tabular}

$\beta$, beta coefficient.

of the four sections and in overall knowledge than the younger age group $[F(1,199)=40 \cdot 65, P=0 \cdot 000]$.

In the most part, knowledge levels varied with education. Individuals who had completed high school or less had significantly less knowledge about food sources of nutrients than those who had technical, trade or university qualifications $[F(2,197)=19 \cdot 28, P=0 \cdot 000]$. Overall, individuals who had a tertiary degree had the highest levels of knowledge $[F(2,197)=21 \cdot 78, P=0 \cdot 000]$. Employment status also influenced knowledge levels; those who were employed in some capacity (part time or full time) had the highest level of knowledge and students had the lowest.

Respondents who were currently or previously married or living as married had significantly higher levels of knowledge than single people across all four sections and overall $[F(1,199)=21 \cdot 95, P=0 \cdot 000]$. Parents of two or more children had greater knowledge of food sources of nutrients and the diet-disease relationships, and significantly greater levels of overall nutrition knowledge.

SES, represented by SEIFA, appeared to influence levels of knowledge. While there was no significant difference in participants' knowledge of the nutrition recommendations, individuals from the higher SES area scored significantly higher for the other three sections of knowledge and the mean overall knowledge score was 12 points greater than that for the lower SES sample $[F(1,199)=23 \cdot 59, P=0 \cdot 000]$.

\section{Multivariate analysis}

To determine the independent effects of each variable on the knowledge level, multiple regression analysis was used. The significant predictors from the univariate analysis were entered into the model. Results show that age, employment status, highest level of education and gender had significant independent effects on nutrition knowledge level (at the 0.05 level). The model accounts for $40 \%$ of the variance in nutrition knowledge scores (Table 2).

\section{Discussion}

\section{General nutrition knowledge}

The results of the present study provide insight into the level of understanding of nutrition information within a South Australian community sample. Due to the nature of recruitment, the sample is not representative but was biased towards females. Given that previous studies have shown females have greater nutrition knowledge levels than males ${ }^{(12,17,20-22)}$, the results of the present study could possibly overestimate the 'true' level of nutrition knowledge throughout the wider South Australian community. Knowledge levels reported in the present study may overestimate community knowledge levels up to twofold, as national data suggest $32 \%$ of people are aware of the recommended vegetable intake ${ }^{(17)}$ compared to $62 \%$ reported in the present study. While recruitment aimed to get a representative sample from two differing SES areas, due to the nature of volunteering, caution should be exercised if generalising beyond this sample. The results can still be valuable in highlighting sections of the community with low levels of knowledge to which nutrition education programmes are to be targeted. The nutrition messages included in education programmes targeting these sub-sections of the community may be different from those of different levels of knowledge. Collecting nationally representative data would be useful for the development of future national nutrition campaigns.

It appears that the key dietary guidelines, like eating more fruit and vegetables and less fatty foods, are reaching the community but detailed knowledge of the nutrient content of foods and converting knowledge to food choice is poor. For example, the knowledge to eat more vegetables is good but how many servings is recommended is less well understood. Knowledge to reduce fat intake is good but knowledge of the energy density of fat, the type of fat to cut down on and the type of foods low or high in fat is poor. Targeting these specific areas of knowledge within the community needs further work.

Knowledge of diet-disease relationships has received much research attention ${ }^{(18)}$, but community knowledge of these relationships is poor and has not improved for many years ${ }^{(15,19)}$. The relationship between fat intake and disease is best understood; yet, one in five people are still oblivious to any relationship. This is alarming as messages about dietary fat are long standing relative to other nutrition messages and the relationship with heart disease is almost axiomatic, yet still parts of the community are unaware. Nutrition educators need to find innovative ways to get these messages across to 
all sections of the community and increase the awareness of the role of diet in disease prevention and health promotion.

The media is one of the most important sources of nutrition information ${ }^{(26,27)}$ and misinformation in the community, but there is a need for health promoters to develop simple and consistent messages. Recent media attention in Australia has favoured high-protein lowcarbohydrate diets and recognition of this information appears to be foremost in the community. This media attention has created uncertainty as to the healthiness of carbohydrate-rich foods despite the fact that a highcarbohydrate low-fat dietary approach underlies the Australian public health nutrition guidelines.

There is evidence that a well-designed campaign promoting nutrition messages like the Go for $2 \& 5$ campaign can be effective. Using mass media outlets and a consistent simple nutrition message, this campaign was successful in increasing community awareness about nutrition recommendations and changing behaviour. Despite scientific papers showing otherwise ${ }^{(28)}$, there is strong public opinion 'that experts never agree about what foods are good for you $^{\text {(12) }}$ and thus it is imperative that nutrition educators and policy makers promote consistent and concise nutrition messages, via high-impact media outlets.

\section{Demographic variation in knowledge}

The demographic variation in nutrition knowledge levels reported in the present study is consistent with other findings. Sub-samples of the community with the lowest levels of knowledge include those residing in lower SES areas, the unemployed and less educated and males. This is of concern as some of these groups are at increased risk of diet-related conditions ${ }^{(29)}$.

Accepting nutrition knowledge has some influence on dietary behaviour; the findings of the present study can be useful in a number of ways. Firstly, health promotion campaigns can be targeted at those population groups most at risk of lower knowledge levels. If deficiencies in knowledge contribute to deficiencies in the diet then education campaigns could be helpful in improving the dietary intake of targeted community groups. Secondly, future nutrition education programmes can aspire to correct consumer misinformation and focus resources on sections of nutrition knowledge that are most poorly understood. This gain in knowledge could influence food choice and dietary intake at a population level.

The present study does stimulate thought as to the type and level of nutrition knowledge required by the community. What level of knowledge is needed to initiate positive changes in dietary behaviour and facilitate a healthy dietary intake to reduce disease risk? Addressing this question is outside the scope of the present study but future research could explore this and other domains of nutrition knowledge, which may be influential in facilitating behaviour change.
The primary objective of nutrition education programmes is to promote healthy dietary behaviour through heightened awareness of nutrition concepts and an increased knowledge of the composition of healthy foods ${ }^{(20)}$. Increased nutrition knowledge is not sufficient for behaviour change, but is necessary ${ }^{(14)}$. Social marketing techniques could be used to increase the awareness of simple public health nutrition messages to initiate positive behaviour changes on a large-scale community level.

\section{Acknowledgements}

Sources of funding: G.A.H.'s $\mathrm{PhD}$ candidature is jointly funded by Flinders University and CSIRO Human Nutrition.

Conflicts of interest: There are no conflicts of interest.

Authorship responsibilities: G.A.H. collected the data, analysed the data, wrote the initial manuscript and made revisions. J.C. and D.C. supervised G.A.H. and reviewed the manuscript.

\section{References}

1. Bandura A (1986) Social Foundations of Thought and Action: A Social Cognitive Theory. New Jersey: Prentice-Hall International Inc.

2. Axelson ML \& Brinberg D (1992) The measurement and conceptualization of nutrition knowledge. J Nutr Educ 24, 239-246.

3. Axelson ML, Federline TL \& Brinberg D (1985) A metaanalysis of food- and nutrition-related research. J Nutr Educ 17, 51-54.

4. Levy AS, Fein SB \& Stephenson M (1993) Nutrition knowledge levels about dietary fats and cholesterol: 1983-1988. J Nutr Educ 25, 60-66.

5. Main FAM \& Wise A (2002) Relationship between knowledge and claimed compliance with genuine and false nutrition messages. J Hum Nutr Diet 15, 349-353.

6. Turrell G \& Kavanagh AM (2006) Socio-economic pathways to diet: modelling the association between socioeconomic position and food purchasing behaviour. Public Health Nutr 9, 375-383.

7. Kristal AR, Bowen DJ, Curry SJ, Shattuck AL \& Henry HJ (1990) Nutrition knowledge, attitudes and perceived norms as correlates of selecting low-fat diets. Health Educ Res $\mathbf{5}$, 467-477.

8. Dallongeville J, Marecaux N, Cottel D, Bingham A \& Amouyel P (2000) Association between nutrition knowledge and nutritional intake in middle-aged men from Northern France. Public Health Nutr 4, 27-33.

9. Wardle J, Parmenter K \& Waller J (2000) Nutrition knowledge and food intake. Appetite 34, 269-275.

10. van Dillen SME, Hiddink GJ, Koelen MA, de Graaf C \& van Woerkum CMJ (2008) Exploration of possible correlates of nutrition awareness and the relationship with nutritionrelated behaviours: results of a consumer study. Public Health Nutr 11, 478-485.

11. Ball K, Crawford D \& Mishra GD (2006) Socio-economic inequalities in women's fruit and vegetable intakes: a multilevel study of individual, social and environmental mediators. Public Health Nutr 9, 623-630.

12. Hansbro J, Bridgwood A, Morgan A \& Hickman M (1997) Health in England 1996: What People Know, What People Think, What People Do: A Survey of Adults Aged 16-74 in England Carried Out by Social Survey Division of ONS on 
Behalf of the Health Education Authority. London: The Stationery Office.

13. Klohe-Lehman DM, Freeland-Graves J, Anderson ER, McDowell T, Clarke KK, Hanss-Nuss H, Cai G, Puri d \& Milani TJ (2006) Nutrition knowledge is associated with greater weight loss in obese and overweight low-income mothers. J Am Dietetic Assoc 106, 65-75.

14. Worsley A (2002) Nutrition knowledge and food consumption: can nutrition knowledge change food behaviour? Asia Pacific J Clin Nutr 11, Suppl., S579-S585.

15. Crawford DA \& Baghurst KI (1990) Diet and health: a national survey of beliefs, behaviours and barriers to change in the community. Aust J Nutr Diet 47, 97-104.

16. Pollard CM, Miller MR, Daly AM, Crouchley KE, Donoghue $\mathrm{KJ}$, Lang AJ \& Binns CW (2008) Increasing fruit and vegetable consumption: success of the Western Australian Go for $2 \& 5^{(\mathrm{R})}$ campaign. Public Health Nutr 11, 314-320.

17. Woolcott Research Pty Ltd (2007) Evaluation of the National Go for $2 \& 5$ Campaign. Prepared for: Australian Government Department of Health and Ageing. New South Wales: Australian Government Department of Health and Ageing.

18. Sapp SG \& Jensen HH (1997) Reliability and validity of nutrition knowledge and diet-health awareness tests developed from the 1989-1991 Diet and Health Knowledge Surveys. J Nutr Educ 29, 63-72.

19. Parmenter K, Waller J \& Wardle J (2000) Demographic variation in nutrition knowledge in England. Health Educ Res 15, 163-174.

20. Variyam JN, Blaylock J \& Smallwood DM (1996) Modelling nutrition knowledge, attitudes, and diet-disease awareness: the case of dietary fibre. Stat Med 15, 23-35.
21. Frank E, Winkleby M, Fortmann SP \& Farquhar JW (1993) Cardiovascular disease risk factors: improvements in knowledge and behaviour in the 1980's. Am J Public Health 83, 590-593.

22. Cotugna N, Subar AF, Heimendinger J \& Kahle L (1992) Nutrition and cancer prevention knowledge, beliefs, attitudes, and practices: The 1987 National Health Interview Survey. J Am Diet Assoc 92, 963-968.

23. Australian Bureau of Statistics (2004) Technical Paper Census of Population and Housing: Socio-Economic Indexes for Areas (SEIFA) Australia 2001. Canberra: Australian Bureau of Statistics.

24. Hendrie GA, Cox DN \& Coveney J (2008) Validation of the General Nutrition Knowledge Questionnaire in an Australian community sample. Nutr Diet 65, 72-77.

25. Parmenter K \& Wardle J (1999) Development of a general nutrition knowledge questionnaire for adults. Eur J Clin Nutr 53, 298-308.

26. Keenan DP, AbuSabha R \& Robinson NG (2002) Consumer understanding of the Dietary Guidelines for Americans: insights into the future. Health Educ Behav 29, 124-135.

27. Fernandez-Celemin L \& Jung A (2006) What should be the role of the media in nutrition communication? BrJ Nutr $\mathbf{9 6}$, S86-S88.

28. Cannon G (1992) Food and Health: The Experts Agree. An Analysis of One Hundred Authoritative Scientific Reports on Food, Nutrition and Public Health Published Throughout the World in Thirty Years, Between 1961 and 1991. London: Consumers' Association Ltd.

29. Australian Bureau of Statistics (2004-2005) National Health Survey: Summary of Results. Canberra: Australian Bureau of Statistics. 